\title{
Efficacy of Turkish isolate of Steinernema feltiae (Rhabditida: Steinernematidae) in controlling the Mediterranean fruit fly, Ceratitis capitata (Wiedemann) (Diptera: Tephritidae), under laboratory conditions
}

\author{
Sabrina Chergui ${ }^{1 *}$ (D) Abdelmdjid Benzehra ${ }^{1}$, Khadidja Boudjemaa ${ }^{1}$, Hadjer Barkou ${ }^{1}$ and Ismail Karaca²
}

\begin{abstract}
The Mediterranean fruit fly, Ceratitis capitata (Wiedemann) (Diptera: Tephritidae), is one of the most destructive pests in fruit growings. It pupates in the soil. The pupae are target of many organisms sheltering the soil such as the entomopathogenic nematodes (EPN). Pathogenicity of the Turkish strain of the EPN, Steinernema feltiae, was evaluated against late instar larvae, pupae, and adults of $C$. capitata under laboratory conditions. Suspensions of the nematode were applied at four increasing concentrations of (0 (for control) 50, 100, and $200 \mathrm{IJs} / \mathrm{ml}$ ) in $1 \mathrm{ml}$ of distilled water. The infectivity of $S$. feltiae against soil stages of C. capitata under different soil moisture levels of 100, 75 , and $50 \%$ of field capacity was evaluated. Mortality rates were recorded after 5 days of treatment. In order to confirm the nematode infection, the dead larvae and pupae were collected and incubated until the appearance of the infectious juvenile (IJS) or dissected under a stereomicroscope to check for nematodes. The last instar larvae and newly formed pupae were more susceptible to EPN infection than old pupae. The infectivity was directly proportional to the increase of soil moisture. The highest mortality (75\%) was obtained. S. feltiae was able to infect adults easily because of the multiple ways of entrance for the nematodes (mouth, anus, and spiracles) than the larvae and/or pupae. Therefore, the Turkish isolate of $S$. feltiae could be useful for an integrated pest management program of $C$. capitata.
\end{abstract}

Keywords: Ceratitis capitata, Steinernema feltiae, Nematode density, Soil moisture

\section{Background}

The Mediterranean fruit fly, Ceratitis capitata (Wiedemann) (Diptera: Tephritidae), is one of the most dangerous pests of fruit crops in the Mediterranean region. The use of insecticides as the sole way of combating this pest has caused environmental pollution and represents a risk for humans and animals in addition to the resistance that has appeared in the insect. The difficulty of controlling this pest's larvae, especially by beneficial insects, comes from the hiding of the larvae inside the infested fruits far from the parasitoids and/or the predators. However, after going through two molts,

\footnotetext{
* Correspondence: chergui.sabrina92@gmail.com

'Département de Zoologie Agricole et Forestière, Ecole Nationale Supérieure

d'Agronomie, El-Harrach, Algeria

Full list of author information is available at the end of the article
}

these larvae leave the fruit by a characteristic jump, sink a few centimeters deep into the soil, and pupate to become the target of entomopathogenic nematodes (EPN). After locating a host, infectious juvenile stages (IJs) penetrate into the host through natural orifices or the cuticle of the insect (Peters and Ehlers 1994) and release their symbiotic bacteria into the hemocoel. The host is quickly killed by sepsis.

EPNs of the genera Steinernema and Heterorhabditis are widely studied, so far about 90 species of Steinernematidae and 20 species of Heterorhabditidae have been described (Labaude and Griffin 2018); however, only few species are commercially produced for use in biological control (Lacey et al. 2015) mainly Steinernema carpocapsae, S. feltiae, and Heterorhabditis bacteriophora. These EPNs are widely used to control insect pests with life 
stages in the soil (Grewal et al. 2005). Several entomopathogens have shown great efficacy in controlling the populations of the first and second larval instars than the third instar of different scarab grub species. The combination of EPN with entomopathogenic fungi optimizes their efficacy against these stages (Laznik and Trdan 2015).

Susceptibility varies with EPN species and the stage of the host development. Indeed, insect larvae are often more susceptible to EPN infection than adults (Odendaal et al. 2016). Trdan et al. (2009) indicated that both the temperature and the developmental stage of the pest have an important influence on the efficacy of EPN as pest-control agent. The mortality of Bactrocera dorsalis induced by Heterorhabditis taysearae ranged from 51.2 to $96.1 \%$ depending on isolates, despite the fact that all isolates were from Benin (Godjo et al. 2018).

Laboratory bioassays are important and allow selecting the most virulent species and isolates of EPN. Steinernema feltiae was tested against several pests of different orders including Coleoptera, Diptera, and Hemiptera. Previous research on the biology and the ecology of EPNs made better forecasts of their performance in the field. Indeed, environmental parameters such as temperature, moisture, vegetation types, and soil properties can affect the survival and virulence of nematodes, while infection depends on interactions between IJs, their symbiotic bacteria and the host (Labaude and Griffin 2018). In this regard, several researches are carried out to increase the efficiency thanks to the selection of the strains and the improvement of production methods (Testa and Shields 2017), formulation (Kim et al. 2015) and application (Bai et al. 2016).

It is in this context, the present study aimed at evaluate the pathogenicity of the Turkish isolate of $S$. feltiae against immature stages of $C$. capitata under laboratory conditions.

\section{Materials and methods Target pest}

C. capitata larvae and pupae were obtained from the mass-rearing unit of the Plant Protection Department, Suleyman Demirel University, Turkey. The colony was maintained under controlled conditions at $25 \pm 1{ }^{\circ} \mathrm{C}, 65 \%$ $\mathrm{RH}$, under 14:10 (L:D) photoperiod. Larvae were reared in sterile Petri dishes containing artificial diet of: water (56 $\mathrm{ml})$, sugar $(12 \mathrm{~g}), \mathrm{Hcl}(0.3 \mathrm{ml})$, wheat germ $(4 \mathrm{~g})$, yeast $(3 \mathrm{~g})$, Benzoic acid $(0.3 \mathrm{~g})$, and bran $(23 \mathrm{~g})$. Adults were provided by water and a solid diet consisting of sucrose and yeast.

\section{Entomopathogenic nematode}

Bioassays were carried out with Turkish commercial strain of S. feltiae (Nematac 10 million) obtained from BioGlobal Campany (Antalya). Aqueous suspensions of nematodes were prepared at different concentrations of $0,50,100$, or $200 \mathrm{IJs} / \mathrm{ml}$.

\section{Susceptibility of Ceratitis capitata soil stages to entomopathogenic nematodes}

The bioassays took place in a controlled environment room at $25 \pm 1{ }^{\circ} \mathrm{C}, 62 \pm 5 \% \mathrm{RH}$ and 16:8 (L:D) photoperiod. Pupae and third instar larvae used for these experiments were collected from the artificial rearing. Effectiveness of the Turkish strain of S. feltiae in controlling soil stages of $C$. capitata was evaluated by exposing individuals to different concentrations of nematode suspensions (0 (C0), $50(\mathrm{C} 1), 100(\mathrm{C} 2)$, or 200 (C3) IJs/ml). These concentrations were chosen starting from the recommended commercial application of EPNs $\left(2.5-5 \times 10^{9}\right.$ $\mathrm{IJs} / \mathrm{ha}=25-50 \mathrm{IJs} / \mathrm{cm}^{2}$ ) (Georgis and Hague 1991). Four replicates with 25 individuals were tested for each treatment. In the control treatment, $1 \mathrm{ml}$ of distilled water without nematodes (D0) was applied. The dead individuals were dissected under a stereomicroscope to determine if the nematodes were present.

\section{Contact method}

Appropriate amounts of nematode concentrations ( 0,50 , 100 , or $200 \mathrm{IJ} / \mathrm{ml}$ ) were counted under a stereomicroscope and added to a filter paper with $1 \mathrm{ml}$ of distilled water in a 9-cm Petri dish (Mahmoud 2007). All concentrations were tested on filter paper against the third instar C. capitata larvae and pupae in four replicates. Twenty-five individuals of each were exposed for $24 \mathrm{~h}$ to each concentration. Controls were treated with $1 \mathrm{ml}$ of distilled water without nematodes. Mortality percentages were recorded for larvae at $24 \mathrm{~h}$ after treatment and after 15 days post-treatment for pupae to record emergence rate and pupal mortality.

\section{Soil method}

Infection took place in plastic cups $(9 \mathrm{~cm}$ diameter and $5 \mathrm{~cm}$ deep) containing $50 \mathrm{~g}$ of natural sieved soil at $10 \%$ of moisture. EPNs were applied on the soil surface at the concentrations of $0,50,100$, and $200 \mathrm{IJs}$ in $1 \mathrm{ml}$ of distilled water. Controls were sprayed by $1 \mathrm{ml}$ of distilled water without nematodes. One hour after treatment, 25 newly formed pupae (0.0-24 h old) and 25 6-day-old ones of C. capitata were placed on the treated soil surface, where 25 third instar medfly larvae were placed on the soil surface in each cup and were left to move into the soil. These cups were covered by a lid and placed in a closed plastic container. Pupation of full-grown larvae took place within $6-10 \mathrm{~h} ; 48 \mathrm{~h}$ after nematode treatment, the soil in each cup was sieved to obtain C. capitata larvae and pupae. Pupae were monitored daily for a period of 15 days for the emergence of adults. Dead pupae and larvae were dissected under a stereomicroscope to confirm the presence of EPN inside. 


\section{Susceptibility of $C$. capitata adults to entompathogenic nematodes}

One millimeter suspension of S. feltiae at different concentrations of 50,100, or 200 IJs was mixed with $1 \mathrm{ml}$ of $10 \%$ sugar solution for adults provided with a piece of cotton. Flies consumed the processed diet within 2-3 days of treatment. At the same time, a nematode-free diet was provided to adults used as controls. The adults were placed in $(10 \times 20 \times 15 \mathrm{~cm})$ cages under rearing conditions. Each treatment was repeated four times. Adult mortality was recorded 5 days after treatment.

\section{Influence of soil moisture on Steinernema feltiae infectivity to $C$. capitata soil stages}

The efficiency of S. feltiae against C. capitata larvae and pupae was investigated under three levels of relative soil moisture (100, 75, and 50\% of soil field capacity). First, a soil sample was sent to the Laboratory of Soil Sciences, Suleyman Demriel University, in order to determine the soil field capacity and moisture. The field capacity in the soil samples used in the experiment was determined as $28.96 \%$ soil moisture. Therefore, in the treatment at $100 \%$ of field capacity, soil moisture was standardized at $28.96 \%$ in the $75 \%$ treatment, moisture was standardized at $21.72 \%$; and in the $50 \%$ treatment, moisture was standardized at $14.48 \%$. The bioassay was carried out according to the experimental procedure and was maintained in an incubator at $25 \pm 1{ }^{\circ} \mathrm{C}, 70$ $\pm 10 \% \mathrm{RH}$, and a 12 -h photophase. A completely randomized experimental design was used with four replicates.

\section{Influence of soil moisture on Steinernema feltiae infectivity to $C$. capitata larvae and pupae}

The efficiency of $S$. feltiae against larvae and pupae of $C$. capitata was studied under three different soil moistures in a completely randomized design with four replicates each. Ten larvae and pupae of $C$. capitata were transferred to plastic pots $(12 \mathrm{~cm} \times 6 \mathrm{~cm})$ containing $100 \mathrm{~g}$ of soil treated with an aqueous suspension of $100 \mathrm{IJs} / \mathrm{ml}$. The control treatment received $2 \mathrm{ml}$ of distilled water without nematodes. The plastic pots were covered and maintained in incubators at $25 \pm 1{ }^{\circ} \mathrm{C}, 70 \pm 10 \% \mathrm{RH}$, and a 12-h photophase. Mortality rates were recorded after 5 days of treatment.

\section{Statistical analysis}

Mortality rates were corrected according to Abbott's formula (Abbott 1925). One-way ANONA was used to compare the mortality of $C$. capitata. Means were compared at the $P=0.05$ level, and Tukey's test was used to separate means (Prism 7).

\section{Results and discussion}

Pathogenicity of the Turkish strain of the EPN, S. feltiae against last instar larvae, pupae, and adults of C. capitata at 4 different concentrations of $0,50,100$, or $200 \mathrm{IJs} / \mathrm{ml}$ was evaluated under laboratory conditions.

\section{Susceptibility of C. capitata larvae}

Results indicated that in both treatments, soil application and contact method, all concentrations caused higher cumulative mortality than the control treated with $\mathrm{C} 0$, where no infection was observed $(F=32.53$, $\mathrm{DF}=2, P<0.0001)$. However, the Tukey test revealed no difference between the two treatment methods (Fig. 1).

Infection of $C$. capitata larvae by $S$. feltiae occurred in a very short time. Eighty-two percent of mortality in 24 $\mathrm{h}$ post-nematode treatment in larvae treated with the highest concentration $\mathrm{C} 3$ for the treatment in the soil against $69 \%$ of mortality recorded in larvae treated at the same concentration in contact method.

Results in Fig. 2 show that mortality rates increased with the increase of nematode concentration with a shock effect obtained in $24 \mathrm{~h}$ post treatment with $\mathrm{C} 2$ and $\mathrm{C} 3$ causing respectively 54 and $82 \%$ mortality in the treatment carried out in the soil and 56 and $69 \%$ mortality in the contact treatment carried out on a filter paper. In fact, the first signs of nematode infection appeared in the first hour after treatment, when a color change in the larvae was noticed. The first dead individuals were recorded 6 hrs post treatment. Dead larvae were dissected under a stereomicroscope in order to confirm the infection by the EPN (Fig. 3).

Larvae escaped from infection developed to pupae but some of them died as pupae. The monitoring of these pupae until emergence revealed a very significant difference in the emergence rate in treated larvae than the control $(F=43.91, \mathrm{DF}=2, P<0.0001)$. Obtained observation is consistent with other reports that most EPN-infected larvae of C. capitata and other tephritids died after forming puparia (Sirjani et al. 2009). In

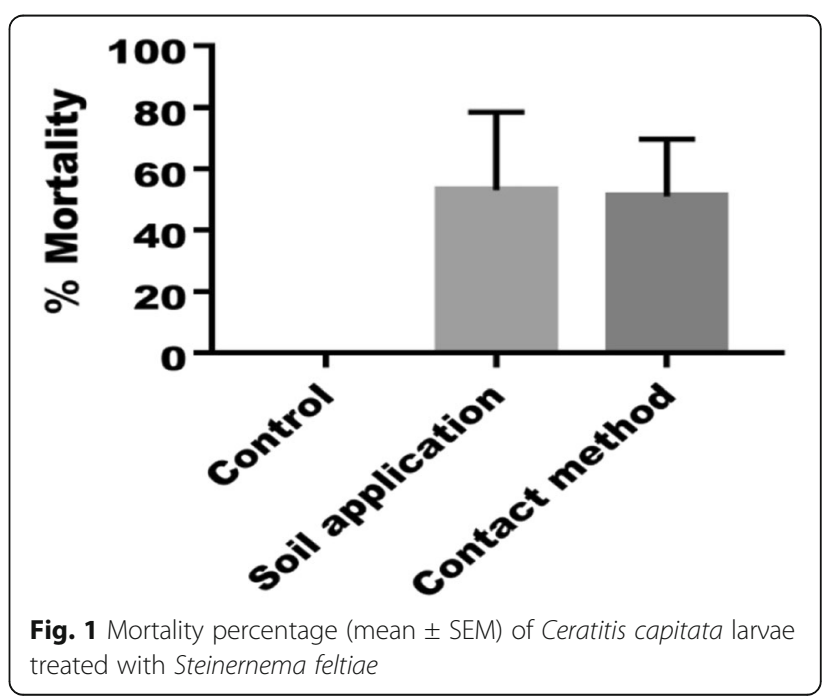


A

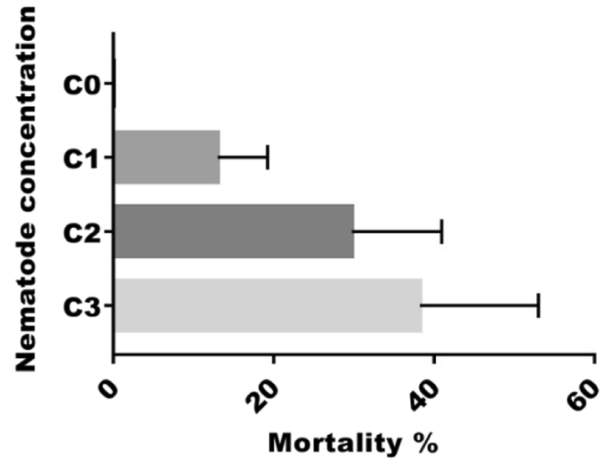

B

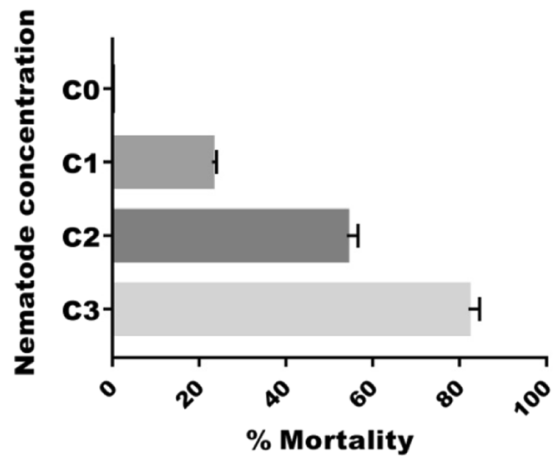

Fig. 2 Mortality percentage (mean \pm SEM) of Ceratitis capitata larvae treated with different concentration of Steinernema feltiae. a Soil application. b Filter paper application

another study, the pathogenicity of a Turkish strain of $S$. feltiae (09-31) showed that the majority of medfly larvae were killed before they could form puparia. These data suggested that S. feltiae (09-31) Aydin isolate was highly virulent to medfly larvae (Karagoz et al. 2009) and may support the conclusion that this species is adapted to dipterous larvae (Lewis et al. 2006). In an additional treatment, $S$. feltiae had an effect on the emergenced flies of $C$. capitata. We recorded 11.76 and $11.23 \%$ of adult mortality from larvae treated in soil and filter paper, respectively.

\section{Susceptibility of C. capitata pupae}

Treatment with EPNs led to the mortality of pupae of $C$. capitata and resulted in a decrease in pupal emergence rate than the untreated ones $(F=21.17, \mathrm{DF}=2, P<0.0001)$. However, older pupae and newly formed ones did not respond in the same way to treatment. Indeed, the statistical analysis revealed significant differences between the mortality rate for young and older pupae $(F=94.11$, $\mathrm{DF}=2, P<0.0001)$. The Tukey test showed that the sensitivity of young pupae to nematodes was greater than that of older ones. In fact, mortality was high in newly formed pupae than older pupae and with a response concentration-related mortality increased as inoculation rate of nematodes increased $(F=187.2$, $\mathrm{FD}=3, P<0.0001)$. According to Mahmoud and Osman (2007), the pathogenicity of $S$. feltiae against Bactrocera zonata (Diptera: Tephritidae) caused a high mortality reaching a rate of $32 \%$ for pupae of 4 days old and 20\% for pupae of 6 days old. In a study on Ragholetis indifferens pupae, Yee and Lacey (2003) justified that the EPNs had probably penetrated intersegmental membranes before the last sclerotization of the integuments.

Despite low percentages of mortality occurred in old pupae of C. capitata (6 days); mortality in emerged flies from survived pupae however was high. A mortality rate of $10.19 \%$ in adults from young treated pupae for nematode suspensions and $37.04 \%$ mortality in adults from older pupae. These adults are probably infected from the soil during the emergence from the pupae. Uncompleted emergences and dead adults showed wings that were not fully spread and juvenile nematodes in the entire body.

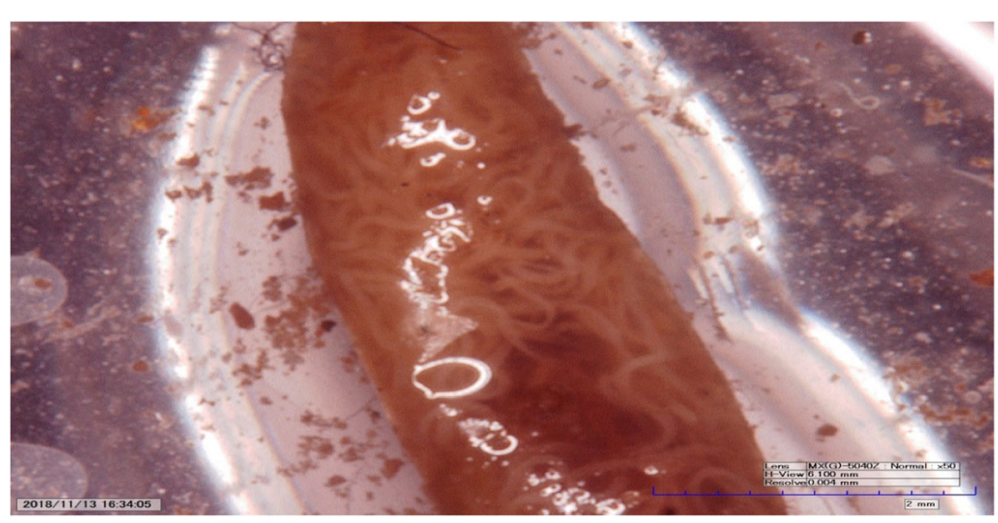

Fig. 3 Last instar larvae of Ceratitis capitata parasitized by Steinernema feltiae seen under a stereomicroscope 
Williams et al. (2015) stated that Heterorhabditis downesi or S. carpocapsae infected pine weevils (Hylobius abietis), the insects died from emergence into adulthood, suggesting that nematode juveniles can infect pupae and survive metamorphosis of their host and adults.

\section{Susceptibility of $C$. capitata adults}

S. feltiae was effective and very virulent on C. capitata adults (Fig. 4). The application of nematode suspension caused higher cumulative mortality than the control treated with concentration $\mathrm{C} 0$, where no infection was observed $(F=219.8, \mathrm{DF}=3, P<0.0001)$. The treatment with nematode suspension at concentration $\mathrm{C} 2$ and $\mathrm{C} 3$ caused (54 and 69\%), respectively. The mean mortality percentage increased in a parallel manner with the increase in EPN concentrations.

The results revealed the great pathogenicity of the Turkish strain of S. feltiae against C. capitata. However, susceptibility of different stages of $C$. capitata was different; larvae and newly formed pupae were more susceptible to nematode infection than old pupae ( $>48 \mathrm{~h}$ ). Obtained results are consistent with several studies conducted on Tephritid flies and indicated that larvae are the most susceptible stage to EPNs infection (Yee and Lacey 2003; Kamali et al. 2013; Nouh and Hussein 2014 and Shaurub et al. 2015). Kamali et al. (2013) explained reasons for high incidence of infection of larvae compared to pupae and adults are due to their developmental duration, activity in soil, output of cues related to host finding by EPNs and larger natural openings. For example, the low susceptibility of pupae, which is highly observed in other studies (Hübner et al. 2017) can be attributed to lack of natural pathway entry for nematodes, as well as a more tough cuticle (Garriga et al. 2018). In fact, the large natural openings of the body of the larvae and the weakly sclerotized larval integument (relative to the nymphal integument) facilitate infection by the

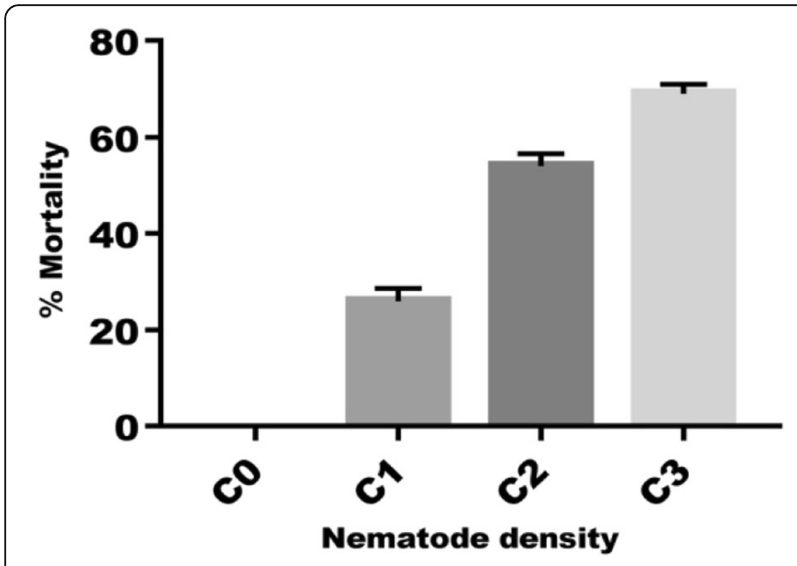

Fig. 4 Mortality percentage (mean \pm SEM) of Ceratitis capitata adults treated with different concentrations of Steinernema feltiae nematode. Labaude and Griffin (2018) justified these differences in susceptibility by various mechanisms, such as differences in behavior, especially high activity levels and avoidance behaviors in adults, as well as more potent immune system or physical barriers to penetration of nematodes. Thus, the highest susceptibility of larvae to EPNs may be related to a greater locomotion at this stage, with greater release of $\mathrm{CO}_{2}$, a chemical compound that plays a role in the attraction of the EPNs (Yee and Lacey 2003).

\section{Influence of soil moisture on S. feltiae infectivity to $C$. capitata soil stages}

The infectivity of S. feltiae to larvae and pupae of C. capitata was determined, under laboratory conditions for different soil moisture levels of 100,75 , and $50 \%$ of field capacity. According to the results illustrated in Fig. 5, the soil moisture level had an effect on the infectivity of $\mathrm{S}$. feltiae to C. capitata soil stages. A highly significant difference was recorded between the control and the batch of larvae treated at different moisture levels $(F=41.32$, DF $=$ $3, P<0.0001)$ and $(F=19.1, \mathrm{DF}=3, P<0.0001)$ for pupae.

The efficiency of nematodes was similar at 100 and $50 \%$ of field capacity, but at $75 \%$ of field capacity, nematodes were more effective against the two soil stages of $C$. capitata. In fact, $S$. feltiae induced a great host mortality when soil moisture was at $75 \%$ of field capacity, causing respectivement (82 and 38\%) of mortality in larvae and pupae, with low efficiency in the other treatments (100 and 50\%). No mortality was observed in the control.

Environmental parameters such as temperature, humidity, vegetation types, and soil properties can affect the survival and virulence of nematodes. Shaurub et al. (2015) indicated that nematode infectivity decreased with increase in exposure time to UV light, whereas it increased with increase in temperature. Infectivity increased in sandy soil, whereas it decreased in silt and clay soils. Soil moisture plays a key role in the mobility of nematode infective juveniles and thus their ability to search for and infect a host. Several studies indicated that soil moisture influence infectivity of EPNs, demonstrating, in general, a decrease in infectivity as soil moisture decreases (Grant and Villani 2003 and Alekseev et al. 2006) and many studies reported low nematode infectivity in extreme, low, and high soil moistures (near the saturation point) (Koppenhöfer et al. 1995).

Glazer (2002) demonstrated that the low infectivity at the highest moisture can be explained by the fact that soil saturation with water reduces oxygen concentration and restricts nematode mobility, which is required to infect the host; however, the low infectivity of nematodes at the lowest moisture content is probably related to the lack of water between the pores, which is also limiting for nematode locomotion. Another possibility for the 

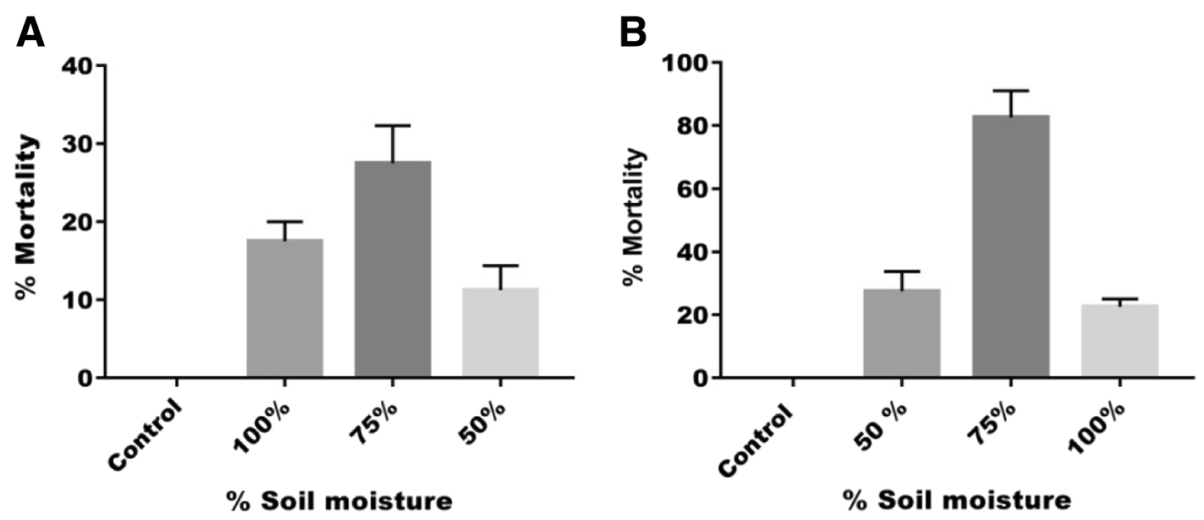

Fig. 5 Mean mortality ( \pm SEM) of Ceratitis capitata pupae (a) and larvae (b) inoculated with Steinernema feltiae at a rate of $100 \mathrm{IJs} / \mathrm{ml}$ and maintained under different levels of soil moisture

lowest infectivity at the lowest moisture content is that these nematodes have developed physiological and behavioral adaptations that allow them to reduce their metabolism, in case of dehydration entering a state of anhydrobiosis (Glazer 2002). Anhydrobiosis can be reversed by wetting the soil, causing a recovery of nematode infectivity and virulence. Studies have demonstrated that some species of the Steinernema have the ability to enter a state of anhydrobiosis, when exposed to low moisture contents (Koppenhöfer et al. 1995), but nothing is clear on this issue regarding Heterorhabditis spp. Since adequate humidity is essential for the survival and movement of this nematode species (Baimey et al. 2015 and Filgueiras et al. 2016).

\section{Conclusion}

Future work should focus on selecting more EPN species/strains to select the most virulent for field trials. In addition, their biology (persistence, reproduction, survival) post-application of EPNs should be studied, as well as their performance in combination with other biological agents and agrochemicals. Although it is often difficult to control moisture in the field, one solution is the addition of adjuvants or the use of a surfactant in the suspension of nematodes that are used as a biocontrol agent. The Turkish isolate of S. feltiae may be an early solution for an integrated pest management program for certain dipteran including C. capitata.

\section{Abbreviations}

C.capitata: Ceratitis capitata; EPN: Entomopathogenic nematodes;

S.feltiae: Steinernema feltiae

\section{Acknowledgements}

Not applicable

\section{Authors' contributions}

SC carried out the experiment and was a major contributor in writing the manuscript. $A B$ conceived and planned the experiments. $K B$ carried out the experiment. $\mathrm{HB}$ corrected and revised the manuscript, corrected language mistakes and translation, and corrected references. IK supervised the work. All authors read and approved the final manuscript

\section{Authors' information}

IK is a professor Dr at Faculty of Agriculture Department of Plant Protection, Süleyman Demirel University. AB is a professor Dr at Ecole Nnationale Sperieure d'Agronomie, El_Harrach, Algeria. SC is Phd student at Zoologie Agricole et Forestière, ENSA, Algeria

\section{Funding}

Not applicable

Availability of data and materials

Not applicable

Ethics approval and consent to participate

Not applicable

\section{Consent for publication}

Not applicable

\section{Competing interests}

The authors declare that they have no competing interests

\section{Author details}

'Département de Zoologie Agricole et Forestière, Ecole Nationale Supérieure d'Agronomie, El-Harrach, Algeria. ${ }^{2}$ Plant Protection Department, Isparta Applied Sciences University, Isparta, Turkey.

Received: 1 May 2019 Accepted: 20 August 2019

Published online: 30 August 2019

\section{References}

Abbott WS (1925) A method of computing the effectiveness of an insecticide. Econ Entomol 18(2):265-267

Alekseev E, Glazer I, Samish M (2006) Effect of soil texture and moisture on the activity of entomopathogenic nematodes against female Boophilus annulatus ticks. BioControl 51(4):507-518

Bai GY, Xu H, Fu YQ, Wang XY, Shen GS, Ma HK, Ruan WB (2016) A comparison of novel entomopathogenic nematode application methods for control of the chive gnat, Bradysia odoriphaga (Diptera: Sciaridae). J Econ Entoml 109(5): 2006-2013

Baimey H, Zadji L, Afouda L, Moens M, Decraemer W (2015) Influence of pesticides, soil temperature and moisture on entomopathogenic nematodes from southern Benin and control of underground termite nest populations. Nematology 17:1057-1069

Filgueiras CC, Willett DS, Junior AM, Pareja M, El Borai F, Dickson DW, Duncan LW (2016) Stimulation of the salicylic acid pathway aboveground recruits entomopathogenic nematodes belowground. PloS One 11(5):e0154712 
Garriga A, Morton A, Garcia-del-Pino F (2018) Is Drosophila suzukii as susceptible to entomopathogenic nematodes as Drosophila melanogaster? J Pest Sci 91(2):789-798

Georgis R, Hague NGM (1991) Nematodes as biological insecticides. Pestic Outlook 2(3):29-32

Glazer I (2002) Survival biology 205-220. In: Gaugler R (ed) Entomopathogenic nematology. Wallingford CABI Publishing UK, p 400

Godjo A, Zadji L, Decraemer W, Willems A, Afouda L (2018) Pathogenicity of indigenous entomopathogenic nematodes from Benin against mango fruit fly (Bactrocera dorsalis) under laboratory conditions. Biol Control 117:68-77

Grant JA, Villani MG (2003) Soil moisture effects on entomopathogenic nematodes. Environ Entomol 32(1):80-87

Grewal PS, Ehlers RU, Shapiro-llan DI (2005) Nematodes as Biocontrol Agents. CABI Pub. pp. 505

Hübner A, Englert C, Herz A (2017) Effect of entomopathogenic nematodes on different developmental stages of Drosophila suzukii in and outside fruits. BioControl 62(5):669-680

Kamali S, Karimi J, Hosseini M, Campos-Herrera R, Duncan LW (2013) Biocontrol potential of the entomopathogenic nematodes Heterorhabditis bacteriophora and Steinernema carpocapsae on cucurbit fly, Dacus ciliatus (Diptera: Tephritidae). Biocontrol Sci Technol 23(11):1307-1323

Karagoz M, Gulcu B, Hazir C, Kaya HK, Hazir S (2009) Biological control potential of Turkish entomopathogenic nematodes against the Mediterranean fruit fly Ceratitis capitata. Phytoparasitica 37(2):153

Kim J, Jaffuel G, Turlings TC (2015) Enhanced alginate capsule properties as a formulation of entomopathogenic nematodes. BioControl 60(4):527-535

Koppenhöfer AM, Kaya HK, Taormino SP (1995) Infectivity of entomopathogenic nematodes (Rhabditida: Steinernematidae) at different soil depths and moistures. J Invertebr Pathol 65(2):193-199

Labaude S, Griffin C (2018) Transmission success of entomopathogenic nematodes used in pest control. Insects 9(2):72

Lacey LA, Grzywacz D, Shapiro-llan DI, Frutos R, Brownbridge M, Goettel MS (2015) Insect pathogens as biological control agents: back to the future. Invertebr Pathol 132:1-41

Laznik Ž, Trdan S (2015) Failure of entomopathogens to control white grubs (Coleoptera: Scarabaeidae). Acta Agric Scand B 65(2):95-108

Lewis EE, Campbell J, Griffin C, Kaya H, Peters A (2006) Behavioral ecology of entomopathogenic nematodes. Biological control 38(1):66-79

Mahmoud MF (2007) Combining the botanical insecticides NSK extract, NeemAzal T 5\%, Neemix 4.5\% and the entomopathogenic nematode Steinernema feltiae Cross N. 33 to control the peach fruit fly, Bactrocera zonata (Saunders). Plant Prot Sci. Institute of Agricultural and Food Information, Prague, Czech Republic 43(1):19-25

Mahmoud MF, Osman MAM (2007) Use of the nematode Steinernema feltiae Cross N 33 as a biological control agent against the Peach Fruit Fly Bactrocera zonata. Tunis J Plant Prot 2:109-115

Nouh GM, Hussein MA (2014) The role of entomopathogenic nematodes as biocontrol agents against some tephritid flies. Adv Biol Res 8(6):301-306

Odendaal D, Addison MF, Malan AP (2016) Control of diapausing codling moth, Cydia pomonella (Lepidoptera: Tortricidae) in wooden fruit bins, using entomopathogenic nematodes (Heterorhabditidae and Steinernematidae). Biocontrol Sci Techn 26(11):1504-1515

Peters A, Ehlers RU (1994) Susceptibility of leatherjackets (Tipula paludosa and Tipula oleracea; Tipulidae; Nematocera) to the entomopathogenic nematode Steinernema feltiae. J Invertebr Pathol 63(2):163-171

Shaurub EH, Soliman NA, Hashem AG, Abdel-Rahman AM (2015) Infectivity of Four Entomopathogenic Nematodes in Relation to Environmental Factors and Their Effects on the Biochemistry of the Medfly Ceratitis capitata (Wied.) (Diptera: Tephritidae). Neotrop Entomol 44:610-618

Sirjani FO, Lewis EE, Kaya HK (2009) Evaluation of entomopathogenic nematodes against the olive fruit fly, Bactrocera oleae (Diptera: Tephritidae). Biol Control 48(3):274-280

Testa AM, Shields EJ (2017) Low labor "in vivo" mass rearing method for entomopathogenic nematodes. Biol control 106:77-82

Trdan S, Vidrih M, Andjus L, Laznik Ž (2009) Activity of four entomopathogenic nematode species against different developmental stages of Colorado potato beetle, Leptinotarsa decemlineata (Coleoptera, Chrysomelidae). Helminthologia 46(1):14-20

Williams CD, Dillon AB, Ennis D, Hennessy R, Griffin CT (2015) Differential susceptibility of pine weevil, Hylobius abietis (Coleoptera: Curculionidae), larvae and pupae to entomopathogenic nematodes and death of adults infected as pupae. Biocontrol 60(4):537-546

Yee WL, Lacey LA (2003) Stage-specific mortality of Rhagoletis indifferens (Diptera: Tephritidae) exposed to three species of Steinernema nematodes. Biological Control 27(3):349-356

\section{Publisher's Note}

Springer Nature remains neutral with regard to jurisdictional claims in published maps and institutional affiliations.

\section{Submit your manuscript to a SpringerOpen ${ }^{\circ}$ journal and benefit from:}

- Convenient online submission

- Rigorous peer review

- Open access: articles freely available online

- High visibility within the field

- Retaining the copyright to your article

Submit your next manuscript at $\boldsymbol{\nabla}$ springeropen.com 